\title{
The microbiome of the human skin and its variability in psoriasis and atopic dermatitis
}

\author{
Małgorzata Mazur ${ }^{1}$, Hanna Tomczak ${ }^{2,3}$, Martha Lodyga ${ }^{1}$, Rafał Czajkowski ${ }^{4}$, Ryszard Żaba ${ }^{2}$, Zygmunt Adamski ${ }^{5}$ \\ ${ }^{1}$ Medical Dermatology Clinic, Poznan, Poland \\ 2Department of Dermatology and Venerology, Poznan University of Medical Sciences, Poznan, Poland \\ ${ }^{3}$ Central Microbiological Laboratory, H. Święcicki Clinical Hospital at the Poznan University of Medical Sciences, Poznan, Poland \\ ${ }^{4}$ Department of Dermatology, Sexually Transmitted Diseases and Immunodermatology, Faculty of Medicine, Nicolaus Copernicus \\ University in Torun, Poland \\ ${ }^{5}$ Department and Clinic of Dermatology, Poznan University of Medical Sciences, Poznan, Poland
}

Adv Dermatol Allergol 2021; XXXVIII (2): 205-209

DOI: https://doi.org/10.5114/ada.2021.106197

\begin{abstract}
The human organism is inhabited by very diverse microorganisms, which constitute the so-called human microbiome and are necessary for the proper functioning of the macroorganism. The correct microbiome ensures homeostasis of the body. A disturbance in its homeostasis leads to dysbiosis. Such deviations may also be related to the development of inflammatory skin diseases, including atopic dermatitis and psoriasis. This review aims to analyse the most current published data on the microbiome of the human skin and examine its role in cutaneous skin diseases, such as atopic dermatitis and psoriasis. This review was compiled by collaborating dermatologists specializing in atopic dermatitis and psoriasis. A comprehensive review of current literature was done using PubMed and limited to relevant case reports and original papers on the skin microbiome in atopic dermatitis and/or psoriasis. It has not been yet established whether changes in the microbiome are the cause or consequence of disease (atopic dermatitis/ psoriasis). However, it was found that in the cases where pathological microflora predominated, an intensification of lesion severity is observed, while with clinical improvement, commensal microflora is restored. Modification of the composition of the microflora may lead to changes in the activation of the immune system and eventually to the development of inflammatory diseases. Adverse effects on the microbiome may include antibiotics, poor diet, stress and adverse environmental conditions. However, more research is needed to identify exact details and mechanisms.
\end{abstract}

Key words: microbiome, atopic dermatitis, psoriasis, dysbiosis.

\section{Introduction}

The human organism is inhabited by very diverse microorganisms, which constitute the so-called human microbiome and are necessary for the proper functioning of the macroorganism [1, 2]. The term "microbes" was first used by Nobel laureate Joshua Lederberg [3]. More and more data on the human microbiome began appearing at the end of the twentieth century, which was associated with the development of molecular techniques that enabled the diagnosis and assessment of the composition of the microbiome: skin, gastrointestinal, respiratory and urogenital tracts [1]. The United States National Institutes of Health $(\mathrm{NIH})$ Common Fund's Human Microbiome Project (HMP), conducted in 2007-2012, provided very detailed data on the human microbiome. The correct microbiome ensures homeostasis of the body. A disturbance in its homeostasis leads to dysbiosis. The topic of dysbiosis is widely analysed in the etiopathogenesis of inflammatory diseases, e.g. non-specific inflammatory bowel diseases. Dysbiosis is associated with increased oxidative stress (including increased production of nitric oxide - NO), damaging DNA repair mechanisms and predisposing to the development of pathological changes. Such deviations may also be related to the development of inflammatory skin diseases, including atopic dermatitis and psoriasis [3].

\section{What is the skin microbiota?}

The skin is colonized by many different microorganisms. Species of these microorganisms and the ratios

Address for correspondence: Hanna Tomczak, Central Microbiological Laboratory, H. Święcicki Clinical Hospital at the Poznan University of Medical Sciences 49 Przybyszdewskiego St, 60-355 Poznan, Poland, phone: +48 509749 200, +48 61 869 13 17, e-mail: hannatomczak@interia.pl Received: 5.02.2020, accepted: 6.05.2020. 
within each cluster are determined by the location, age, occupation, environmental factors, cosmetics applied and the coexistence of other diseases as well as the use of topical drugs [4].

The microbiome of the human skin is a complex ecosystem consisting of commensal, symbiotic microorganisms (bacteria, fungi, viruses, protozoa) that live in homeostasis with the entire organism. It plays an important role in providing protection against pathogenic strains that may be responsible, among others, for skin diseases [5]. In the situation of a disturbance in the composition and functioning of the skin microbiome, there may be increased susceptibility to the development of inflammatory diseases. It is known that the skin microbiome depends on the local "microenvironment" of a given location. In adults, there are differences in the composition and concentration of the bacterial population between sites that are relatively more sebaceous, moist or dry [4]. The least amount of microorganisms can be isolated from areas that are constantly exposed and in contact with environmental factors (e.g. skin of the limbs) - the most prevalent organisms found in these places are Proteobacteria and Flavobacteriales. However, the opposite is observed in intertriginous regions, where there is a moist and relatively warm environment. Under such conditions, Staphylococcus aureus and Corynebacterium spp. are better able to thrive [6]. Alternatively, in areas rich in sebaceous glands one will encounter more lipophilic yeast including Malassezia spp. and Pirynobacterium spp. On the head, including the scalp, nose, ears and hair, Propionibacterium predominate, which is much less seen on the skin of the shoulders [7]. Different hormonal influences in both women and men also determine the dissimilarities between their skin microbiome [8]. Holland and Bojar have stated that even the type of cosmetics used may affect the composition and quality of the skin microbiome [9]. The colonization of the skin surface is also related to oxygen tolerance (aerotolerance), where obligate aerobes or relatively anaerobic (facultative) microorganisms will prevail. In deeper layers where oxygen is restricted, there are obligate anaerobes.

The main bacteria that colonize the skin are Staphylococcus epidermidis and other coagulase-negative staphylococci, as well as bacteria from the Actinobacteria spp., Corynebacterium spp., Propionibacterium spp., Brevibacterium spp., and Micrococcus spp. are other predominant bacteria of the skin, which are considered to be permanent skin microflora. On the other hand, Staphylococcus aureus, Streptococcus pyogenes and Pseudomonas aeruginosa are classified as transient microflora, which are mainly seen during pathological conditions [10]. A study conducted by Iwase et al. showed that S. epidermidis might inhibit $S$. aureus biofilm formation [11]. This is most likely connected with the fact that S. epidermidis, which dominate, are the first to populate the niches and thus, do not allow S. aureus to flourish.
The age of the patient is also an important factor in the diversity of the microbiome composition. The majority of research on skin microbiology focused on the skin of adults, and less attention was given to the skin of children. Foetal skin in the uterus is sterile. Bacterial colonization occurs during delivery. There is a significant difference depending on whether the delivery was a natural birth or caesarean section. The microbiome of the newborn, which came into the world as a result of a natural birth, is similar to the maternal vaginal microbiome, whereas the caesarean-born neonate is colonized with microflora from skin of the mother or the personnel facilitating labour, whichever has the most significant skin-to-skin contact first [12]. However, these initial differences (within $24 \mathrm{~h}$ ) resulting from the mode of childbirth disappear within the first month of life. The way of feeding the newborn also plays a huge role. Along with rapid colonization of the surface, there are changes in the function of the skin barrier (including reduction of transepidermal water loss, skin $\mathrm{pH}$ and sebaceous activity) [13]. As it is known, the skin of an infant differs from that of an adult in structure, function and biochemical composition [14]. Kimberly et al. analysed whether the microbiome of babies' skin differs from that of an adult and whether it evolves with the development of the child's skin. The horny layer (stratum corneum) of the infant's skin is more hydrated than in an adult, that is why the infant's microbiome resembles a more moist area of the skin compared to adults (relatively higher numbers of Staphylococcus spp. in infants) [5].

However, the skin-specific evolution of bacteria begins in the first 3 months of life. Three regions were analysed - buttocks, shoulders and forehead. The number of species in a given area does not change significantly during the first year of life, the relative density of bacteria increased with the age of the child. In contrast to the skin of an adult, in which Proteobacteria, Actinobacteria and Firmicutes predominate, infants are mainly colonized by Firmicutes, followed by Actinobacteria, Proteobacteria and Bacteroidetes [15]. Depending on the location, the greatest amounts of Clostridium bacteria (Phylum firmicutes) occurred on the skin of the buttocks, while Bacilli and Firmicutes were more often isolated on the skin of the arms and forehead. Additionally, it was shown that in the two youngest groups of infants (1-3 months and 4-6 months), Streptococcus spp. and Staphylococcus spp. dominate, which constitute up to $40 \%$ of the total skin microbiome [15]. In the examined children, an increase in Propionibacterium spp. was found in samples from the forehead in the 4-6 months age group, which correlates with the period in which infantile acne begins to appear [16]. The area around the buttocks proved to be most unique in its microflora. The study discovered a kind of Finegoldia - these are the newly described protease-producing commensal bacteria, which due to their abundance on the skin of the buttocks may play a role in the development of irritation 
in the diaper region. Early colonization of the skin of the buttocks by Clostridium spp. and other microorganisms belonging to the gastrointestinal microflora are associated with the proximity of the anus and diaper region. The most important difference between children and adults is that the skin microbiome in adults is stable, while in children it is unstable (because it is just forming), which can create conditions in babies for abnormal skin development and inflammation $[5,15]$.

The microbiome of the skin, in addition to bacteria, also includes viruses, fungi and protozoa. Three dominant virus families have been identified on the skin surface: Papillomaviridae ( $\beta-, \gamma$-HPV), Polyomaviridae and Circoviridae, with the majority of individuals having Papillomaviruses $\beta$ and $\gamma$ [17]. The presence of bacteriophages has been determined, e.g. for Propionibacterium spp., Staphylococcus spp., Pseudomonas spp., on the skin and the number of which varies depending on the location [18].

Most of the fungi on healthy skin are species of the genus Malassezia: Malassezia restricta, Malassezia globosa, Malassezia sympodialis, Malassezia pachydermatis and Malassezia furfur, with Malassezia restricta and Malassezia furfur being the most numerous ones [19]. In a study of 11 body locations (forehead, left and right ear, left and right axillary region, left and right antecubitis, left and right forearm, left and right legs), in eight of the healthy adult participants, Malassezia spp. accounted for about $80 \%$ of all fungi colonizing the skin [20].

In exceptional cases, Candida spp. can colonize the skin, but their presence is more often associated with the occurrence of infections, especially in immunocompromised individuals or in patients with diabetes [21].

\section{Microbiome in atopic dermatitis}

An infant's skin is sensitive and prone to the development of inflammatory changes, including eczematous lesions and diaper dermatitis. The currently observed increase in atopic dermatitis (AD) is of particular importance. Recently, the relationship between clinical manifestations of $A D$ and the skin microflora profile of patients has been analysed. It was found that in the cases where pathological microflora predominated, an intensification of lesion severity is observed, while with clinical improvement, commensal microflora is restored. It is also known that in patients with AD there is a dysfunction of the skin barrier function, which promotes the colonization by Staphylococcus aureus. During exacerbations of atopic dermatitis, microbiome changes involve increased Staphylococcus aureus colonization. In addition, staphylococci modulate inflammatory responses through various mediators and participate in the control of skin inflammation [15]. Immunological pathways in the skin, as in the gut, are associated with the development of allergy or asthma [22]. The evolution of the skin microbiome together with the patient's age can stimulate the maturation of the immune system by providing epitopes for training for the immune system [23]. Some species of bacteria produce proteases that can alter the barrier function of the skin [24]. The microflora may also be affected by the use of topical medicines (corticosteroids or immunomodulators), as well as moisturizing and oiling preparations (emollients) that are used as part of primary or secondary prophylaxis. However, this requires more detailed research with a control group. Generally, it is believed that treatment favouring the growth of symbiotic bacteria can restore the balance of microflora, which plays an important role in the course of AD. The presence of various gram-positive bacteria, including from the genus Lactococcus, Streptococcus and Streptomyces, limits the growth of other pathogenic bacteria, which has beneficial effects on skin lesions. Some emollients contain bacteria that can act on the skin like a probiotic.

\section{Microbiome in psoriasis}

Psoriasis is a chronic, recurrent inflammatory skin disease that occurs with excessive epidermal proliferation and immunologic dysfunction. Factors that may exacerbate the course of psoriasis include infections: bacterial, viral, fungal and parasitic. Already in the 1970s, there was a connection established between streptococcal infection (throat) and the development of disseminating psoriasis. Fry and Baker showed that some bacteria, including Staphylococcus aureus and Streptococcus pyogenes, viruses (human papillomavirus and endogenous retroviruses) and fungi (Malassezia spp. and Candida albicans) can affect the course of the disease [25]. Although further research is required to establish the relationship between the cutaneous microbiome and psoriasis, current research suggests that microbiota in patients with psoriasis differs from healthy controls [26]. Alekseyenko et al. showed that in the psoriatic plaques there are a higher number of bacteria: Corynebacterium spp., Propionibacterium spp., Staphylococcus spp. and Streptococcus spp. than in healthy skin [27]. In the Fahlén et al. study, it was found that the most common bacteria isolated from both lesions and normal skin in psoriatic patients was Streptococci and Firmicutes, whereas in comparison to the microbiome of healthy controls, patients with psoriasis had a smaller number of Staphylococcus spp., Actinobacteria and Propionibacterium spp. [28]. Gao et al. also showed that Propionibacterium spp. is much less abundant in psoriasis than in the control group [29]. In addition, it was observed that with the improvement after a 3-week balneotherapy, a higher number of Xanthomonadaceae bacteria were found, which belong to the genus Proteobacteria [30]. Also, when analysing the composition of fungi within the microbiome, Malassezia spp. was found to be less frequent in patients with psoriasis than in healthy subjects, with a much greater variety of isolated fungi [31]. However, Gomez-Moyano et al. showed that the increased concentration of Malassezia 
spp. in the skin of patients with psoriasis is observed only in the period of exacerbations [32]. The role of Malassezia spp. in psoriasis may be due to its ability to increase the expression of TGF- $\beta 1$, the integrin chain and HSP70, which stimulates the migration of immune cells and results in the hyperproliferation of keratinocytes [33]. Amongst the entire Malassezia spp., the most isolated species is Malassezia restricta [34]. Interestingly, it was shown that in the circumstances of high concentrations of Malassezia spp. within psoriatic lesions in patients, there is a greater tendency to irritation after treatment with topical calcipotriol (analogue of vitamin D) [35]. Additionally, it has been demonstrated that general antifungal drugs have beneficial effects on the condition of nail plates in the course of psoriasis, which may indirectly explain the role of fungi in the severity of lesions [36]. Attention was paid to the advantageous role of phototherapy, which, by improving the oxidative stress parameter, causes significant changes in the microbiota of the skin. It would be interesting to investigate the relationship between clinical improvement of psoriasis, changes in skin microbiology and systemic oxidative stress parameters after NB-UVB therapy [37].

\section{Conclusions}

The microbiome of the skin is an interesting topic that is more and more noticed and becoming the centre of attention for studies investigating its connection and influence on the human immune system. It have not been yet established whether changes in the microbiome are the cause or consequence of disease (atopic dermatitis/psoriasis). However, the interaction between microflora and the immune system is certainly important for maintaining host homeostasis. Mechanisms related to this role, however, remain unexplained; it is probably related to the inhibition of the growth of pathogenic microorganisms and the strengthening of the host's innate immunity [38]. Modification of the composition of the microflora may lead to changes in the activation of the immune system and eventually to the development of inflammatory diseases. There are hypotheses that the skin microbiome is closely related to the intestinal microbiome. Modification of the microbiome can be achieved through diet and probiotics. Adverse effects on the microbiome may include antibiotics, poor diet, stress and adverse environmental conditions. However, more research is needed to identify exact details and mechanisms.

\section{Conflict of interest}

The authors declare no conflict of interest.

\section{References}

1. Malinowska M, Tokarz-Deptuła B, Deptuła W. Mikrobiom człowieka. Post Microbiol 2017; 56: 33-42.
2. Hooper LV, Gordon JI. Commensal host-bacterial relationships in the gut. Science 2001; 292: 1115-8.

3. Tian T, Wang Z, Zhang J. Pathomechanisms of oxidative stress in inflammatory bowel disease and potential antioxidant therapies. Oxid Med Cell Longev 2017; 2017: 4535194.

4. Grice EA, Segre JA. The skin microbiome. Nature Rev Microbiol 2011; 9: 244-53.

5. Grice EA, Kong HH, Conlan S, et al. Topographical and temporal diversity of the human skin microbiome. Science 2009; 324: 1190-3.

6. Grice EA, Kong HH, Conlan S, et al. Topographical and temporal diversity of the human skin microbiome. Science 2009; 324: 1190-2.

7. Costello EK, Laauber CL, Hamady M, et al. Bacterial community variation in human body habitats across space and time. Science 2009; 326: 1694-7.

8. Marples RR. Sex, constancy, and skin bacteria. Arch Dermatol Res 1982; 272: 317-20.

9. Holland KT, Bojar RA. Cosmetics: what is their influence on the skin microflora? Am J Clin Dermatol 2002; 3: 445-9.

10. Gao Z, Tseng C, Pei Z, Blaser MJ. Molecular analysis of human forearm superficial skin bacterial biota. Proc Natl Acad Sci USA 2007; 104: 2927-32.

11. Iwase T, Uehara Y, Shinji H, et al. Staphylococcus epidermidis spp. inhibits Staphylococcus aureus biofilm formation and nasal colonization. Nature 2010; 465: 346-9.

12. Dominguez-Bello MG, Costello EK, Contreras M, et al. Delivery mode shapes the acquisition and structure of the initial microbiota across multiple body habitats in newborns. Proc Natl Acad Sci USA 2010; 107: 11971-5.

13. Chiou YB, Blume-Peytavi U. Stratum corneum maturation. A review of neonatal skin function. Skin Pharmacol Physiol 2004; 17: 57-66.

14. Stamatas GN, Nikolovski J, Luedtke MA, et al. Infant skin micro-structure assessed in vivo differs from adult skin in organization and at the cellular level. Pediatr Dermatol 2010; 27: 125-31.

15. Capone KA, Dowd SE, Stamatas GN, Nikolovski J. Diversity of the human skin microbiome early in life. J Invest Dermatol 2011; 131: 2026-32.

16. Hello M, Prey S, Leaute-Labreze C, et al. Infantile acne: a retrospective study of 16 cases. Pediatr Dermatol 2008; 25: 434-8.

17. Lecuit M, Eloit M. The human virome: new tools and concept. Trends Microbiol 2013; 21: 510-5.

18. Hannigan GD, Meisel JS, Tyldsley AS, et al. The human skin double-stranded DNA virome: topographical and temporal, genetic enrichment and dynamic associations with the host microbiome. MBio 2015; 6: e01578-15.

19. Paulino LC, Tseng CT, Strober BE, Blaser MJ. Molecular analysis of fungal microbiota in samples from healthy human skin and psoriatic lesion. J Clin Microbiol 2006; 44: 2933-41.

20. Gao Z, Perez-Perez GI, Chen Y, Blaser MJ. Quantitation of major human cutaneous bacterial and fungal populations. I Clin Microbiol 2010; 48: 3575-81.

21. Roth RR, James WD. Microbial ecology of the skin. Annu Rev Microbiol 1988; 42: 444-64.

22. Callard RE, Harper JI. The skin barrier, atopic dermatitis and allergy: a role for Langerhans cells? Trends Immunol 2007; 28: 294-8.

23. Marchini G, Nelson A, Edner J, et al. Erythema toxicum neonatorum isan innate immune response to commensal microbes penetrated into the skin of the newborn infant. Pediatr Res 2005; 58: 613-6. 
24. Hirasawa Y, Takai T, Nakamura T, et al. Staphylococcus aureus extracellular protease causes epidermal barrier dysfunction. J Invest Dermatol 2010; 130: 614-7.

25. Fry L, Baker BS. Triggering psoriasis: the role of infections and medications. Clin Dermatol 2007; 25: 606-5.

26. Benhadou F, Mintoff D, Schnebert B, Thio BH. Psoriasis and microbiota: a systematic review. Diseases 2018; 6: 47.

27. Alekseyenko AV, Perez-Perez GI, De Souza A, et al. Community differentiation of the cutaneous microbiota in psoriasis. Microbiome 2013; 1: 31.

28. Fahlén A, Engstrand L, Baker BS, et al. Comparison of bacterial microbiota in skin biopsies from normal and psoriatic skin. Arch Dermatol Res 2012; 304: 15-22.

29. Gao Z, Tseng CH, Strober BE, et al. Substantial alterations of the cutaneous bacterial biota in psoriatic lesions. PLoS One 2008; 3: e2719.

30. Martin R, Henley JB, Sarrazin P, Seité S. Skin microbiome in patients with psoriasis before and after balneotherapy at the thermal care center of La Roche-Posay. I Drugs Dermatol 2015; 14: 1400-5.

31. Takemoto A, Cho O, Morohoshi Y, et al. Molecular characterization of the skin fungal microbiome in patients with psoriasis. J Dermatol 2015; 42: 166-70.

32. Gomez-Moyano E, Crespo-Erchiga V, Martínez-Pilar L, et al. Do Malassezia species play a role in exacerbation of scalp psoriasis? J Mycol Med 2014; 24: 87-92.

33. Baroni A, Paoletti I, Ruocco E, et al. Possible role of Malassezia furfur in psoriasis: Modulation of TGF- $\beta 1$, integrin, and HSP70 expression in human keratinocytes and in the skin of psoriasis-affected patients. J Cutaneous Pathol 2004; 31: 35-42.

34. Amaya M, Tajima M, Okubo Y, et al. Molecular analysis of Malassezia microflora in the lesional skin of psoriasis patients. J Dermatol 2007; 34: 619-24.

35. Rosenberg EW, Belew PW. Improvement of psoriasis of the scalp with ketoconazole. Arch Dermatol 1982; 118: 370-1.

36. Kaminsky A. Comparative effects of griseofulvin on onychomycosis and on psoriasis unguium. AMA Arch Dermatol 1960; 81: 838-40.

37. Darlenski R, Hristakieva E, Aydin U, et al. Epidermal barrier and oxidative stress parameters improve during in $311 \mathrm{~nm}$ narrow band UVB phototherapy of plaque type psoriasis. J Dermatol Sci 2018; 91: 28-34.

38. Sanford JA, Gallo RL. Functions of the skin microbiota in health and disease. Semin Immunol 2013; 25: 370-7. 